
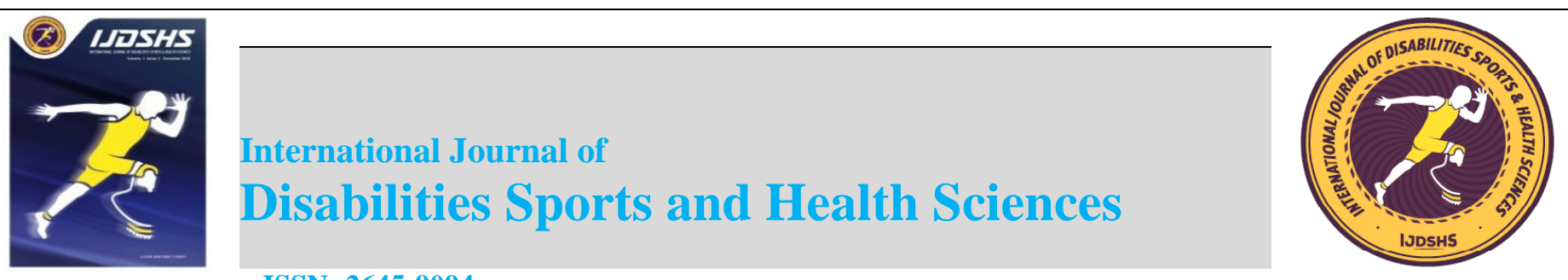

\title{
Effects of kinesio taping on upper extremity functionality in patients with cerebral palsy: a randomized controlled trial
}

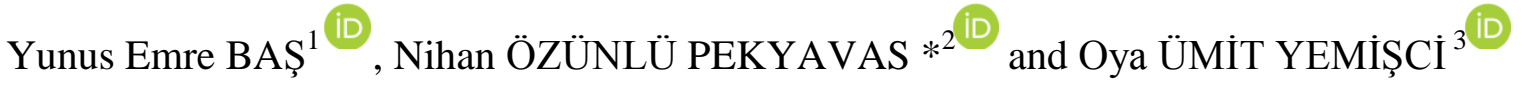 \\ ${ }^{1}$ Uzman Paylaşım Special Education and Rehabilitation Center, Ankara, Turkey \\ ${ }^{*}$ Baskent University, Faculty of Health Sciences, Department of Physiotherapy and Rehabilitation, Ankara, Turkey \\ ${ }^{3}$ Baskent University, Faculty of Medicine, Department of Physical Therapy and Rehabilitation, Ankara, Turkey \\ *Corresponding author: nihan_2002@hotmail.com
}

\begin{abstract}
The aim of this study was to investigate the effects of kinesio taping application on upper extremity functionality in patients with CP. This study included 40 children aged 6-16 years, diagnosed with CP. The children with CP were divided into 2 groups, the study group and the control group. Ligth touch Kinesio taping was applied to study group to improve posture and function in shoulder area. Assessments were done three times for each group (baseline - 45min later - 1 week later). Frenchay Arm Test and Moberg Pick-Up Test were used to evaluate upper extremity functionality in participants with CP. In the control group, no significant difference was found ( $p>0.05$ ). The level of change in Frenchay Arm Test and Moberg Pick-Up Test measurement results performed in different times was found significant in study group $(\mathrm{p}<0.05)$. As a result of the application of kinesio taping for functional correction of the upper extremity in children with $\mathrm{CP}$, an improvement in fine motor functions was revealed. This is one of the first studies giving evidence about the positive effects of pediatric kinesio taping application on functionality in children with $\mathrm{CP}$.
\end{abstract}

Keywords

Cerebral palsy, Kinesio Taping, Upper Extremity, Functionality, Pediatric Rehabilitation

\section{INTRODUCTION}

Cerebral palsy $(\mathrm{CP})$ is the most common cause of physical disability in childhood. Immature brain is a neurodevelopmental disorder caused by non-progressive lesions leading to spasticity, muscle weakness, decreased selective motor control, and secondary musculoskeletal problems often accompanied by sensory and cognitive disorders (Bax et al, 2005).

Hand is one of the most affected parts in CP. Effective use of hand function for daily activities depends on the complex interaction between fine motor functions and perceptions of tactile, proprioceptive and visual information / accuracy (Kase, Wallis, and Kase, 2003). The main prerequisites for this are (a) independent control capacity on fingers, (b) a complex somatosensory system to direct finger movements, and (c) conversion ability (Cepeda, 2008). Primitive and autonomic movements become more evident due to lesions in the sensory motor cortex and corticospinal system, which play a key role in sensitive grip and finger movements in CP. Tonus disorder leads to abnormal movements (loss of motor control, contraction, mutual inhibition) and limitation of movement. As a result of these, children with CP develop dysfunction in fine motor skills (Cepeda, 2008; Kase, Martin, and Yasukawa, 2006).

There are many treatment approaches (neurodevelopmental therapy, sensory integration, targeted training and hand-arm bimanual therapy) and kinesio taping to ensure the development of fine motor skills in children with CP (Cepeda, 2008; Kase, Martin, and Yasukawa, 2006) Kinesio 
taping helps stimulate cutaneous receptors and mechanoreceptors and helps muscle excitability (Roy, 2018; Mohamed, 2016). Kinesio taping technique can be used to increase sensory stimulation, strengthen weak muscles, inhibit spastic muscles, increase joint stability, increase functional motor skills, assist postural control, and improve functional independence in pediatric rehabilitation clinics in addition to other therapeutic techniques (Kase, Martin, and Yasukawa, 2006).

Kinesio taping application, together with other rehabilitation programs used in children with $\mathrm{CP}$, affects the sensorimotor system positively and improves voluntary control and coordination of the upper extremities (Kase, Martin, and Yasukawa, 2006; Chitaria et al., 2015; Yasukawa, Patel, and Sisung, 2006). The aim of this study is to investigate the effects of Kinesio taping on upper extremity functionality in participants with $\mathrm{CP}$.

\section{MATERIALS AND METHODS}

\section{Participants:}

Necessary permission and approval was obtained from the University Ethical Research Ethics Committee for the study to be carried out (Number: KA20/46). The clinical trial number for this study is NCT04529486.

This study included children diagnosed with $\mathrm{CP}$ by a physician and children between 6 and 16 years old. Both genders were included in our study. According to the Gross Motor Function Classification System, 14 (35\%) children were 3rd level $26(65 \%)$ children were at the 2 nd level. None of the participants had spasticity including upper extremity. The study consists of a total of 40 participants with CP. Before the study, the families of children with $\mathrm{CP}$ were informed about the purpose of the study, all applications and evaluations during the study and they were informed about the results and benefits of these applications. "Informed Consent Form" was signed to the families of children with $\mathrm{CP}$ who agreed to participate in the study voluntarily with their consent.

Inclusion criteria were as follows;

- To have a cognitive level to comprehend the instructions

- Not having a cooperative problem that may prevent communication
- Continuing physical therapy and special education regularly

Exclusion criteria were as follows;

- Those with orthopedic problems

- Any surgical procedure

- Participants with cognitive problems

- Participants who do not regularly receive physiotherapy

The children with CP included in this study consisted of 2 groups, the study group $(n=20)$ and the control group $(n=20)$. No application was made to the control group. The assessments were done three times for each group (baseline - 45min later 1 week later) (Figure 1). An experienced physiotherapist carried out all assessments and another experienced physiotherapist made the applications in order to provide a single blinded study. The participants were randomly allocated to the groups by computer randomization.

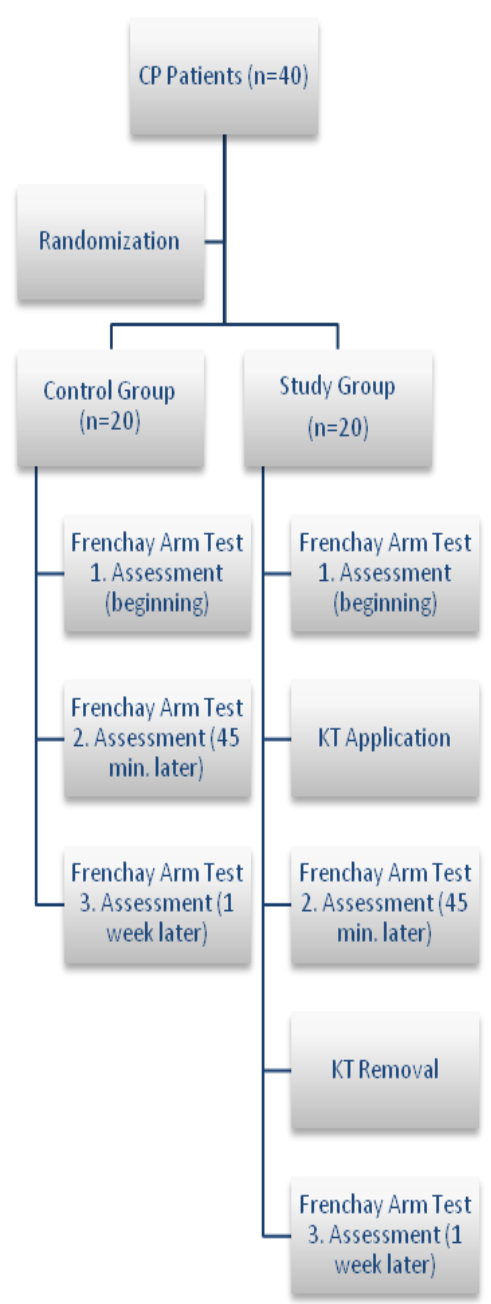

Figure 1. Flow chart of the study

\section{Assessments:}

Socio-demographic and clinical information of each child was obtained from their families and 
hospital files before detailed evaluations within the scope of the study were carried out. An experienced physiotherapist carried out all assessments. The evaluation was carried out in a quiet and calm environment in order to eliminate the factors that may affect the evaluation results such as difficulty of concentration and distraction in children.

Frenchay Arm Test and Moberg Pick-Up Test were used to evaluate upper extremity functionality in participants with $\mathrm{CP}$.

\section{Frenchay Arm Test:}

The total duration of the test is 3 minutes. Each task is scored as 1 point. The participant sits in the chair with a table in front of him and puts his hands on his knees and tries to perform the following sequential tasks with the affected arm / hand.

1. Fixing the ruler: the participant holds the ruler on one sheet of paper with the affected hand and draws a straight line with the other hand. To be considered successful, the ruler must be firmly and properly fixed.

2. Holding a roller: Grasps a $12 \mathrm{~mm}$ diameter and $5 \mathrm{~cm}$ long roller while standing about $15 \mathrm{~cm}$ in the middle of the table edge, lifts it about $30 \mathrm{~cm}$ and releases it without dropping it.

3. Raising the cup: It takes a half-full glass of water, standing at a distance of $15-30 \mathrm{~cm}$ from the edge of the table, drinks a little water and leaves it in place without spilling any water.

4. Attaching a latch to the bar: A wooden stick with a diameter of $10 \mathrm{~mm}, 15 \mathrm{~cm}$ long, inserted into the hole in the middle, is placed $15-30 \mathrm{~cm}$ away from the edge of a 10-square square wooden table. A latch is attached to the bar in the middle of the board. The participant is asked to open the latch with the affected hand and remove it from the rod and then put it back on. During testing, it should not drop the latch and hit the rod.

5. Combing hair: combs the hair with the affected hand (or pretends to be combing the hair). The comb should be held at the top of the head and the back and both sides should be combed. The test is found reliable and valid $(\mathrm{r}=0.68-0.99)$ (Poole \& Whitney, 2001).

\section{Moberg Pick-Up Test:}

Required test objects; 50 cents coin, wing nut screw, 6-point screw nut, 4-point screw nut, 1lira coin, key, screw, screw washer, nail, paper clip, safety pin, large 6-point nut, chronometer, small box, sleep glasses.
The child sits on a chair and is asked to put the objects one by one in the middle box as fast as he can. The child is told not to take the object by sliding it to the corner of the table. When ready, the test is started and the time is recorded with the stopwatch. The test is performed with the dominant hand. Test is repeated three times, recording the best time. The application is found valid and reliable (Moberg, 1958).

\section{Treatment:}

Kinesio taping was applied to the study group to improve posture and improve function in the shoulder area. Measurements were carried out for the study group before and after application (with tape on). The tape was then removed and the measurements were repeated after 1 week. Before applying the tape, it was checked whether the children were allergic. Tape was first started from the distal part of the fingers and taped with 0\% tension with Kinesio Taping Functional Correction Technique. Another I tape was applied from palmar area of the hand to spina scapula for supination of the forearm and external rotation of the shoulder with $\% 0$ tension to make functional correction (Figure 2). Kinesio Taping Functional Correction method is suggested to be done with $75 \%$ of tension but for children, we applied it with $0 \%$ tension to minimize the shear effects to the skin (Kase, 2006). Kinesio Tex Ligth Touch Plus tape was used for taping application (Kinesio Co., USA).

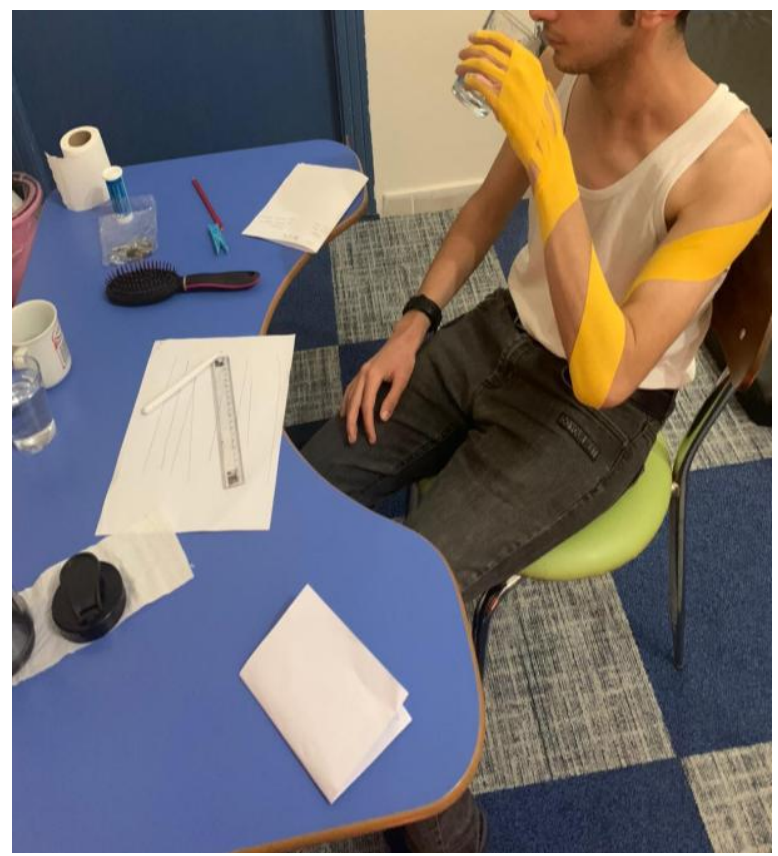

Figure 2. Frenchay Arm Test application with Kinesio taping. 


\section{Data Analysis}

The power analysis indicated that 20 participants for each group were needed with $80 \%$ functionality as the primary outcome. The data were analyzed using statistical software (SPSS version 18, Inc., Chicago, IL, USA). All the statistical analyses were set a priori at an alpha level of $p<0.05$. The tests for homogeneity (Levene's test) and normality (Shapiro-Wilk) were used to determine the appropriate statistical methods to apply for comparison between the groups. If parametric conditions are provided in the evaluation of intra-group differences (change between 3 time factors), two-way analysis of variance is used in repeated measurements, and ttest is used to evaluate differences between groups. The effect size was evaluated to determine the effectiveness of the intervention in the study group. For the effect size value, it is accepted as 0,1-0,3 "low", 0,3-0,5 "medium" and > 0,5 "high". power and a $5 \%$ type 1 error. The power analysis of our study showed a power of $80 \%$ with

\section{RESULTS}

A total of 40 cases, 10 girls and 30 boys, aged 6-16 years, who were voluntarily accepted to participate in the study, were diagnosed with $\mathrm{CP}$, were evaluated. It was observed that the cases were between $12-80 \mathrm{~kg}$ and their height was between $84-181 \mathrm{~cm}$. The majority of children with CP (35.7\%) were found to have an average of $11.36 \pm 3.61$ years of illness in secondary school students. The most common type of spasticity was ataxic spasticity $(35.7 \%)$, and the presence of epilepsy was found in $21.4 \%$ of children with CP. The most common involuntary movements were tremor $(64.3 \%)$, and the most frequently detected pathological reflex was hypotonia (50\%) (Table 1).

Table 1. Sociodemographic characterictics of the participants

\begin{tabular}{|c|c|c|c|}
\hline & Kinesio Group $(n=20)$ & Control Group $(n=20)$ & $\mathrm{p}$ \\
\hline & $\mathrm{X} \pm \mathrm{SD}$ & $\mathrm{X} \pm \mathrm{SD}$ & \\
\hline Age (year) & $11.29 \pm 3.71$ & $12,24 \pm 2.98$ & 0.865 \\
\hline Body Weigth (kg) & $32.46 \pm 18.87$ & $32.61 \pm 16.97$ & 0.923 \\
\hline$\overline{\text { Heigth }(\mathbf{c m})}$ & $124.29 \pm 31.34$ & $123.59 \pm 32.21$ & 0.982 \\
\hline \multicolumn{4}{|l|}{ Gender } \\
\hline Female (n) & 10 & 11 & 0.954 \\
\hline Male (n) & 10 & 9 & \\
\hline$\overline{\text { CP Type }}$ & & & $0.034^{*}$ \\
\hline $\operatorname{Ataxic}(\mathbf{n})$ & 6 & 12 & \\
\hline Dyskinetic (n) & 14 & 8 & \\
\hline
\end{tabular}

*p $<0.05, \mathrm{X} \pm \mathrm{SD}=\mathrm{Mean} \pm$ Standard Deviation, Min-Max=Minimum-Maximum

In the control group, the level of change in the Frenchay Arm Test and Moberg Pick-Up Test measurement values performed at the 3 time factors was found to be statistically insignificant ( $\mathrm{p}$ $>0.05)$. The effect size of the Frenchay Arm Test and Moberg Pick-Up Test applied to the control group over time was found to be low $(0.071$; 0.067) (Table 2).

In kinesio group, the level of change in the Frenchay Arm Test and Moberg Pick-Up Test measurement values performed at the 3 time factors was found to be statistically significant $(p<0.05)$. The effect size of the Frenchay Arm Test and Moberg Pick-Up Test applied to the control group over time was found to be high $(0.831$; 0.862) (Table 2).

Considering the effect of kinesio taping application on upper extremity functionality, both tests were found significantly different in 45 minutes and 1 week after application in kinesio group $(\mathrm{p}<0.05)$. No significant difference was found between $45 \mathrm{~min}$. and 1 week measurements (p>0.05) (Table 3). 
Table 2. The change in groups for upper extremity functionality tests

\begin{tabular}{|c|c|c|c|c|c|c|}
\hline & 1. Assessment & $\begin{array}{l}\text { 2. Assessment } \\
\text { (45 min. later) }\end{array}$ & $\begin{array}{l}\text { 3. Assessment } \\
\text { (1 week later) }\end{array}$ & $\mathrm{p}$ & $\mathrm{Eta}^{2}$ \\
\hline & & $\begin{array}{l}\mathrm{X} \pm \mathrm{SD} \text { (Median; Min- } \\
\text { Max) }\end{array}$ & $\begin{array}{l}\mathrm{X} \pm \mathrm{SD} \text { (Median; Min- } \\
\text { Max) }\end{array}$ & $\begin{array}{l}\mathrm{X} \pm \mathrm{SD} \text { (Median; Min- } \\
\text { Max) }\end{array}$ & & \\
\hline \multirow{2}{*}{ 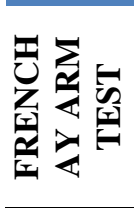 } & $\begin{array}{c}\text { Control Group } \\
(\mathbf{n}=20)\end{array}$ & $2.29 \pm 1.20(2.50 ; 0-4)$ & $2.36 \pm 1.28(2.50 ; 0-4)$ & $2.26 \pm 1.25(2.50 ; 0-4)$ & 0.985 & 0.071 \\
\hline & $\begin{array}{c}\text { Kinesio Group } \\
(\mathbf{n}=\mathbf{2 0})\end{array}$ & $2.39 \pm 1.30(2.40 ; 0-4)$ & $3.50 \pm 1.09(4.0 ; 1-5)$ & $3.29 \pm 1.14(3.0 ; 1-5)$ & $0.18^{*}$ & 0.831 \\
\hline \multirow{2}{*}{ 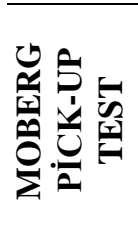 } & $\begin{array}{c}\text { Control Group } \\
(\mathbf{n}=\mathbf{2 0})\end{array}$ & $\begin{array}{c}58.10 \pm 30.34 ;(51.69 \\
22.10-145.17)\end{array}$ & $\begin{array}{c}55.63 \pm 30.61(49.97 \\
19.58-143.17)\end{array}$ & $\begin{array}{c}55.98 \pm 30.86(50.61 \\
20.27-143.52)\end{array}$ & 0.566 & 0.067 \\
\hline & $\begin{array}{c}\text { Kinesio Group } \\
(\mathbf{n}=\mathbf{2 0})\end{array}$ & $\begin{array}{c}57.69 \pm 30.41 ;(51.63 \\
21.14-144.32)\end{array}$ & $\begin{array}{c}47.37 \pm 22.86(43.60 \\
19.02-107.47)\end{array}$ & $\begin{array}{c}48.30 \pm 23.06(44.41 \\
21.57-109.30)\end{array}$ & $0.01 *$ & 0.862 \\
\hline
\end{tabular}

Table 3. The differences between time factors in upper extremity functionality tests

\begin{tabular}{lcccc} 
& \multicolumn{2}{c}{ Control Group (p) } & \multicolumn{2}{c}{ Kinesio Group (p) } \\
\cline { 2 - 5 } & $\begin{array}{c}\text { Frenchay Arm } \\
\text { Test }\end{array}$ & $\begin{array}{c}\text { Moberg Pick-Up } \\
\text { Test }\end{array}$ & $\begin{array}{c}\text { Frenchay Arm } \\
\text { Test }\end{array}$ & Moberg Pick-Up Test \\
\hline $\begin{array}{l}\text { 1. Assessment - 2. Assessment } \\
\text { (45 min. later) }\end{array}$ & 1.00 & 0.96 & $\leq 0.01^{*}$ & $\leq 0.01^{*}$ \\
\hline $\begin{array}{l}\text { 2. Assessment (45 min. later) } \\
\text { - 3. Assessment (1 week later) }\end{array}$ & 1.00 & 0.84 & 0.247 & 0.342 \\
$\begin{array}{l}\text { 1. Assessment -3. Assessment } \\
\text { (1 week later) }\end{array}$ & 1.00 & 0.77 & $\leq 0.01^{*}$ & $\leq 0.01^{*}$ \\
\hline
\end{tabular}

${ }^{*} \mathrm{p}<0.05, \mathrm{~T}$ test, Post-hoc Bonferorroni correction

\section{DISCUSSION}

In this study we investigated the effects of Kinesio Taping on upper extremity functionality in participants with $\mathrm{CP}$, and found that Kinesio taping application may be an effective tool for improving the functional capacity of upper extremities in participants with CP.

In the study of Chitaria et al. (2015), similar to this study, Kinesio Taping method applied on wrist extensor muscles. It has been found that the Clutch and Visual Engine Integration subtest results result in a significant increase. In our study Kinesio Taping Functional Correction method was used to affect functionality of upper extremity in participants with CP. Kinesio Taping Functional Correction method is suggested to be done with $75 \%$ of tension but for children, we applied it with $0 \%$ tension to minimize the shear effects to the skin (Kase, 2006) Kinesio Tex Ligth Touch Plus tape was used for taping application (Kinesio Co., USA). This tape is said to be suitable for pediatric and geriatric populations due to its nanotechnologicproperties(www.kinesiotaping.co m, n.d.).

In their study, Yasukawa et al. (2006) concluded that Kinesio Taping improved kinesthetic inputs and facilitated advanced control of the forearm and wrist muscles to improve voluntary control of muscle and tendon movement during activities, thereby improving hand grip and thus improving hand functions. It can be concluded that extending the tape over the dorsal face of the wrist increases carpal and metacarpal stability, thereby improving intrinsic muscle activity and thus facilitating better finger activities (Kase et al., 2003; Chitaria et al., 2015). Also, taping the dorsal face of the wrist can cause increased stimulation of cutaneous afferents in the underlying skin. The sensorial system provides preliminary information about limb positions and muscle forces to the central nervous system to monitor and control limb movements, plan actions, and provide smooth motion (McGlone \& Reilly, 2010). 
In this study, we think that this technique provides improved stability since Kinesio Taping starts from the hand phalanx and metacarpal bones extends over the forearm and arm muscles and extends to the middle of the scapula behind the shoulder. It can be suggested that this method contributes to the clinical changes in the fine motor functions of the hands. This view is supported by Hsu et al. (2009), who reported that neuromuscular taping, in addition to therapeutic procedures, will lead to improvement in strength, functional activities, proprioception and control. Kinesio Taping improves blood circulation in the taped area (Kase \& Hashimoto, 1998), and this physiological change can affect muscle and myofascial functions following the application of taping and helps children build the strength required for function. In the studies of Roy et al. (2018) in which they evaluated the effect of Kinesio Taping in children with spastic diplegic cerebral palsy, Kinesio Taping has been shown to have a positive effect on enhancing and developing fine motor skills. The conclusion reached by Roy et al. (2018) supports this study.

In a study, it has been reported that muscle patterns can improve due to better proprioception due to stimulation of Kinesio taping mechanoreceptors (Semple et al., 2012). In a study by Demirel and Bayrakc1 (2014) with children with $\mathrm{CP}$, the effect of Kinesio Taping applied to the wrist extensor muscles on the joint range of motion was investigated, and they found an increase in the range of wrist extension, ulnar and radial deviation motion. As a result of this study, it was suggested that Kinesio Taping affects the antagonist muscle of the spastic muscle and may increase the range of motion by removing the fascia. In another study that supports our study; In the studies of Yasukawa et al. (2006) evaluating the application of Kinesio Taping to the upper extremity in children who were admitted to the acute rehabilitation program due to encephalitis, brain tumor, cerebral vascular trauma, traumatic brain and spinal cord injury, the extension and comprehension of the Kinesio Taping, including object manipulation found that functional motor skills increased.

Kinesio Taping stimulates sensory receptors and cutaneous mechanoreceptors in the taped area. Activation of cutaneous mechanoreceptors with a sufficient level of stimulus causes local depolarizations that trigger nerve impulses along the afferent fiber that travels towards the central nervous system (Halseth et al., 2004; Murray \& Husk, 2001). The Kinesio Taping application can exert pressure on the skin or stretch the skin and stimulate cutaneous mechanoreceptors that cause physiological changes in this external load-taped area. Previous studies to determine the effects of neuromuscular taping on cutaneous mechanoreceptors have been shown to increase muscle excitability by taping applied on specific muscles and joints (Halseth et al., 2004; Murray \& Husk, 2001). Effective use of the hand depends on the complex interaction between fine motor skills and visual perception. In the studies of Mohamed (2016) investigating the effect of Kinesio Taping on upper extremity functions in children with hemiplegic $\mathrm{CP}$, it was concluded that this application had a positive effect on wrist motion, grip and visual motor integration. In this study, it was determined that the values obtained as a result of Frenchay Arm Test in children in the study group in which the Kinesio Taping was applied had a significant change in time compared to the initial stage, and this effect showed that the effect size value of this change had a high level of improvement.

Having different ages and disability levels is a limitation of this study. Also including more participants should be considered in future studies. As a result of this study, it has been shown that the application of Kinesio Taping can increase and improve fine motor skills in children with $\mathrm{CP}$, thereby increasing their functional independence to meet the demands of daily life. It can be predicted that this situation will have a positive effect on parents' quality of life. However, Kinesio Taping should be applied by experienced physiotherapists to achieve the desired effectiveness. It can be argued that with the emergence of positive effects of Kinesio Taping application on extremity functionality, the trust of the child and family in the physiotherapist and willingness to work together will increase. Due to its effectiveness, relatively inexpensive cost and easy application, we think that the use of Kinesio Taping application in addition to traditional treatment can help target functional therapy strategies to improve and improve upper extremity functionality in children with CP. However, more comprehensive studies are needed to support its use in clinical practice. 


\section{Conflict of interests}

The authors have no conflict of interests to declare. No financial support was received for this study.

\section{Ethics Statement}

The studies involving human participants were reviewed and approved by the Başkent University Ethical Research Ethics Committee (Date: 3/03/2020; Decision / Protocol number: KA20/46, The clinical trial number for this study is NCT04529486). Written informed consent to participate in this study was provided by the patients/participants.

\section{REFERENCES}

Bax, M., Frcp, D., \& In, R. (2005). Executive committee for the definition of cerebral palsy. Dev Med Child Neurol. 47: 571-6.

Cepeda, J.P., Fishweicher, A., Gleeson, M., Greenwood, S., \& Motyka-Miller, C. (2008). Does Kinesio Taping of the abdominal muscles improve the supine-to-sit transition in children with hypotonia. Retrieved on April, 12, 2011.

Chitaria, S. B., Narayan, A., Ganesan, S., \& Biswas, N. (2015). Short-term effects of kinesiotaping on fine motor function in children with cerebral palsy- a quasiexperimental study. Critical Reviews ${ }^{\mathrm{TM}}$ in Physical and Rehabilitation Medicine, 27(1): 41-50.Cited: 20.08.2020, Available from: www. kinesiotaping.com

Demirel, A., \& Bayrakc1, V. (2014). The effect of kinesio tape on active wrist range of motion in children with cerebral palsy: A pilot study. J Orthop. 6(2).

Halseth, T., McChesney, J. W., DeBeliso, M., Vaughn, R., \& Lien, J. (2004). The effects of kinesio $^{\mathrm{TM}}$ taping on proprioception at the ankle. Journal of sports science \& medicine, 3(1), 1-7.

Henderson, A., \& Pehoski, C. (2006.). Hand function in the child, foundation for remediation. 2nd ed. Philadelphia: Elsevier.

Hsu, Y. H., Chen, W. Y., Lin, H. C., Wang, W. T., \& Shih, Y. F. (2009). The effects of taping on scapular kinematics and muscle performance in baseball players with shoulder impingement syndrome. Journal of electromyography and kinesiology, 19(6), 1092-1099.
Kase, K., \& Hashimoto, T. (1997). Changes in the volume of peripheral blood flow by using kinesio tape. Kinesio Taping Association. 82:1373.

Kase, K., Martin, P., \& Yasukawa A. (2006). Kinesiotaping in pediatrics. Fundamentals and whole body taping. Albuquerque, NM: Kinesio Taping Association.

Kase, K., Wallis, J., \& Kase, T. (2003). Clinical therapeutic applications of the kinesio taping method. 2nd ed. Tokyo, Ken Ikai Co.

McGlone, F., \& Reilly, D. (2010). The cutaneous sensory system. Neuroscience \& Biobehavioral Reviews, 34(2), 148-159.

Moberg, E. (1958). Objective methods for determining the functional value of sensibility in the hand. The Journal of bone and joint surgery. British volume, 40(3), 454476.

Mohamed, N.E. (2016). Kinesio taping for upper limb function in children with hemiplegic cerebral palsy. Int J Adv Res. 4(11):1754-62.

Murray, H., \& Husk, L. (2001). Effect of kinesio taping on proprioception in the ankle. J Orthop Sports Phys Ther. 31:37.

Patel, D.R. (2005). Therapeutic interventions in cerebral palsy. Indian J Pediatr. 72(11):97983.

Poole, J. L., \& Whitney, S. L. (2001). Assessments of motor function post stroke: A review. Physical \& Occupational Therapy in Geriatrics, 19(2), 1-22.

Roy, S., Dixit, J., Kumar, A., \& Singh, O. (2018). The Effect of Kinesiotaping in Improving Fine Motor Skills In Children With Spastic Diplegic Cerebral Palsy. IOSR J Dent Med Sci. 17(11):79-84.

Semple, S., Esterhuysen, C., \& Grace, J. (2012). The effects of kinesio ankle taping on postural stability in semiprofessional rugby union players. Journal of physical therapy science, 24(12), 1239-1242.

Shamsoddini, A., \& Hollisaz, M. T. (2013). Effects of taping on pain, grip strength and wrist extension force in patients with tennis elbow. Trauma monthly, 18(2), 71.

Shamsoddini, A., Hollisaz, M. T., Hafezi, R., \& Amanellahi, A. (2010). Immediate effects of counterforce forearm brace on grip strength and wrist extension force in patients with lateral epicondylosis. Hong Kong Journal of Occupational Therapy, 20(1), 8-12. 
Stamer, M.H. (1996). Posture and movement of the child with cerebral palsy 2nd edition. Texas,USA.

Şimşek, T. T., Türkücüoğlu, B., Çokal, N., Üstünbaş, G., \& Şimşek, İ. E. (2011). The effects of Kinesio ${ }^{\circledR}$ taping on sitting posture, functional independence and gross motor function in children with cerebral palsy. Disability and rehabilitation, 33(21-22), 2058-2063.

Taylor, R. L., O'Brien, L., \& Brown, T. (2014). A scoping review of the use of elastic therapeutic tape for neck or upper extremity conditions. Journal of Hand Therapy, 27(3), 235-246.

Yasukawa, A., Patel, P., \& Sisung, C. (2006). Pilot study: Investigating the effects of Kinesio Taping ${ }^{\circledR}$ in an acute pediatric rehabilitation setting. American Journal of Occupational Therapy, 60(1), 104-110.

How to cite this article: Baş, Y.E., Özünlü Pekyavaş, N. and Ümit Yemişçi, O. (2021). Effects of kinesio taping on upper extremity functionality in patients with cerebral palsy: a randomized controlled trial. Int J Disabil SportsHealth Sci;4(2):152-159.https://doi.Org /10.3343 /ijdshs.1005339 